\title{
Архитектура и программная реализаиия исследовательского стенда корпоративной беспроводной локальной вычислительной сети
}

\author{
А.И. Абросимов 1, д.m.н., профресcop, AbrosimovLI@mpei.ru \\ M.A. Орлова ${ }^{1}$, ассистент, OrlovaMA@треi.ru \\ Х. Хаю 1, аспиранm, hussein.khayou@gmail.com \\ ${ }^{1}$ Наииональный исследовательский университет \\ "Московский энергетический институт", г. Москва, 111250 , Россия
}

В статье представлены архитектура и программная реализация исследовательского стенда для получения и анализа вероятностно-временных характеристик корпоративной беспроводной локальной вычислительной сети (БЛВС). В ходе разработки данного стенда авторами были получены математические соотношения для расчета гарантированной интенсивности мультимедийного трафика.

Исследовательский стенд состоит из двух независимых блоков. Блок «Стенд моделирования» содержит описание БЛВС и потоков мультимедийного трафика в дискретно-событийной системе моделирования ns-3. Блок «Анализ результатов моделирования» содержит программы для анализа файлов передаваемого трафика и результатов моделирования, а также программы расчета характеристик производительности. Блок «Анализ результатов моделирования» написан на языке Python3, анализ файлов передаваемого трафика производился с использованием библиотеки pyshark.

Также в статье содержатся аналитические уравнения модели БЛВС, используемые в блоке «Анализ результатов моделирования». Приведенные уравнения позволяют для заданного времени гарантированной доставки пакетов, для беспроводных каналов связи, использующих заданный канальный протокол, определить предельную интенсивность доставленных пакетов. Программная реализация исследовательского стенда дает возможность получить зависимости гарантированной интенсивности мультимедийного трафика для заданных параметров: структуры БЛВС, параметров беспроводного канала связи и канальных протоколов управления доступом.

Разработанный стенд обеспечивает возможность работы в двух режимах. В режиме разработки новой БЛВС, когда известными параметрами являются паспортные данные оборудования, логические характеристики протоколов и предполагаемые характеристики трафика, используется полный набор функциональных модулей и блоков, стенд позволяет обеспечить как согласование трафика с ресурсами передачи и обработки, так и заданную производительность БЛВС. В режиме эксплуатации, когда мониторинг позволяет получить реальные характеристики трафика и протоколов, стенд дает возможность администратору БЛВС оценить производительность БЛВС и интенсивность трафика. В этом режиме используется ограниченный набор модулей, который требует гораздо меньше времени для оценки производительности БЛВС, предоставляет возможность адаптивно изменять настройки БЛВС и обеспечивать характеристики производительности БЛВС, соответствующие требованиям QoS.

Ключевые слова: программы для моделирования сетей передачи данных, архитектура исследовательского стенда, беспроводные локальные сети, производительность, канальньий протокол.

Целью исследований, результаты которых представлены в настоящей статье, является повышение качества обслуживания мультимедийного трафика и производительности корпоративной беспроводной локальной вычислительной сети (БЛВС) с помощью средств, обеспечивающих согласование интенсивности мультимедийного трафика и производительности БЛВС. Для достижения поставленной цели разработана архитектура исследовательского стенда. Программная реализация позволяет проводить исследование разрабатываемых, а также уже эксплуатируемых БЛВС с помощью определения вероятностно-временных характеристик таких сетей, анализа и аналитических расчетов характеристик производительности.

Авторам неизвестны готовые программные реализации для исследования производительности корпоративных БЛВС, позволяющие настраивать множество параметров беспроводных устройств и трафика, хотя работы в этом направлении ведутся [1-3]. Поэтому потребовалась разработка модели корпоративной БЛВС, а также программ, позволяющих обрабатывать результаты моделирования. Для моделирования корпоративной БЛВС выбрано про- 
граммное средство дискретно-событийного моделирования ns-3 [2], которое широко используется для исследования БЛВС стандарта IEEE 802.11 [4-7]. Ns-3 позволяет создавать модели БЛВС из моделей беспроводных устройств ns-3 (беспроводные станции и точки доступа), которые представляют собой программную реализацию спецификации стандарта IEEE 802.11 для МАС-подуровня и различные модели физического уровня, соответствующие спецификациям стандарта IEEE $802.11 \mathrm{a} / \mathrm{b} / \mathrm{e} / \mathrm{g} / \mathrm{n} / \mathrm{ac} / \mathrm{ax}$ [2].

Таким образом, модели беспроводных устройств поддерживают настройки множества параметров, необходимых для исследования вероятностно-временных характеристик корпоративных БЛВС. Поэтому требуется разработать исследовательский стенд для получения и анализа вероятностно-временных характеристик корпоративной БЛВС. В процессе разработки решены следующие задачи: определены параметры корпоративной БЛВС для получения необходимых характеристик; разработан блок для исследования вероятностно-временных характеристик с помощью дискретно-событийного моделирования; разработаны модули для обработки, анализа полученных характеристик и расчета характеристик производительности БЛВС.

\section{Параметры БЛВС}

Корпоративная БЛВС (рис. 1) содержит точку доступа $\mathrm{AP}, k(k=1, K)$ беспроводных станций STA, сервер (корпоративные и/или мультимедийные интернет-ресурсы (приложения)), коммутационную среду корпоративной сети со скоростью передачи данных не менее 1 Гб/с, представленную как коммутатор.

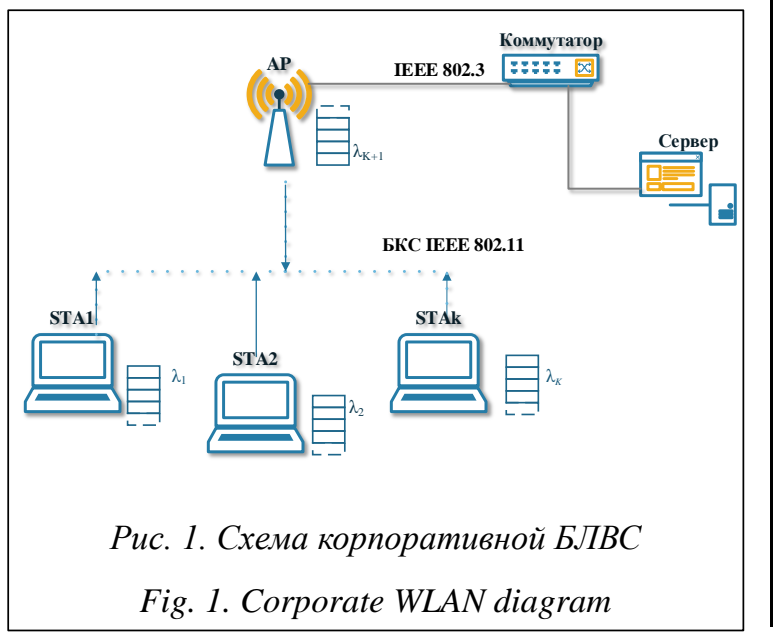

Точка доступа АР и беспроводные станции STA соединены беспроводным каналом связи (БКС) стандарта IEEE 802.11 и образуют инфраструктурный базовый набор обслуживания iBSS (infrastructure Basic Service Set) [8]. Требуется определить параметры корпоративной БЛВС для получения характеристик производительности. Параметры корпоративной БЛВС можно разделить на параметры производительности БЛВС, параметры БКС и параметры потоков трафика сетевых приложений, например, приложений мультимедийного трафика (MMT) [1].

Параметры производительности БЛВС зависят от параметров ее настройки для точки доступа АР и представлены в таблице 1. Одним из таких параметров является dot11BeaconPeriod, который устанавливает период рассылки beacon-кадров, содержащих ряд параметров БЛВС и осуществляющих механизм синхронизации времени между станциями. Для управления доступом к БКС в рассматриваемой БЛВС используется механизм управления доступом CF. Для настройки базовых механизмов CF существуют два канальных протокола (КП) БКС: распределенный DCF и централизованный PCF. Для их настройки используются параметры:

- dot11MediumOccupancyLimit, устанавливающий максимальную продолжительность работы КП РСF;

- dot11CFPPeriod, устанавливающий период переключения из режима работы КП DCF в режим работы КП РСF;

- hw_mode, определяющий стандарт и параметры БКС для установленного стандарта (модуляция, кодирование, частотный диапазон, временные интервалы, метод расширения спектра) [9].

Параметры производительности БКС определяет параметр hw_mode. Основным ресурсом БКС является пропускная способность $C$ [бит/c] (допустимый набор поддерживаемых скоростей передачи данных - параметр basic_rates). Пропускная способность $C$ определяет время $\tau$ передачи одного бита (в сек.): $\tau=1 / C$.

Характеристики производительности БЛВС: время доставки пакета MМТ - $T^{\Phi}{ }_{C F}$, эффективность доставки пакетов с пользованием КП $\mathrm{CF}-U_{C F}$, интенсивность доставленных пакетов - $u_{C F}$, зависят от параметров производительности БЛВС [10], параметров БКС, параметров потоков трафика MMT, а также от 
Параметры производительности БЛВС

Таблицฺа 1

The performance WLAN parameters

Table 1

\begin{tabular}{|c|c|c|}
\hline Параметр & Описание & Допустимые значения \\
\hline dot11BeaconPeriod & Период отправки beacon-кадра & 1..65535 [TU] (1024 us) \\
\hline dot11DTIMPeriod & $\begin{array}{l}\text { Период отправки ТІМ элементов } \\
\text { в beacon-кадре }\end{array}$ & $0 . .255$ [dot11BeaconPeriod] \\
\hline dot11MediumOccupancyLimit & Максимальное время режима РСF & $0 . .1000[\mathrm{TU}]$ \\
\hline dot11CFPPeriod & $\begin{array}{l}\text { Период переключения в режим } \\
\text { РCF в продолжительности DTIM } \\
\text { периода }\end{array}$ & $0 . .255$ [dot11DTIMPeriod] \\
\hline$h w \_m o d e$ & $\begin{array}{l}\text { Режим БКС a = IEEE } 802.11 \mathrm{a} \\
(5 \mathrm{GHz}), \mathrm{b}=\mathrm{IEEE} 802.11 \mathrm{~b}(2.4 \mathrm{GHz}) \text {, } \\
\mathrm{g}=\mathrm{IEEE} 802.11 \mathrm{~g}(2.4 \mathrm{GHz}), \\
\mathrm{ad}=\mathrm{IEEE} 802.11 \mathrm{ad}(60 \mathrm{GHz}) ;\end{array}$ & $\mathrm{a}, \mathrm{b}, \mathrm{g}, \mathrm{ad}$ \\
\hline basic_rates & $\begin{array}{l}\text { Набор поддерживаемых скоро- } \\
\text { стей передачи данных }\end{array}$ & $\begin{array}{l}\text { Зависит от } h w \_m o d e[\mathrm{a}, \mathrm{b}, \mathrm{g}, \mathrm{ad}] \\
\text { например, } h w \_m o d e=\mathrm{g} \\
\text { basic_rates }=(10,20,55,110,60,90 \text {, } \\
120,180,240,360,480,540) \\
\mathrm{kbps}]\end{array}$ \\
\hline$K$ & $\begin{array}{l}\text { Количество беспроводных } \\
\text { станций }\end{array}$ & $1-25$ \\
\hline
\end{tabular}

количества $K$ беспроводных станций, подключенных к точке доступа АР (табл. 1). Параметры потоков трафика ММТ представлены в таблице 2.

\section{Параметры потоков трафика ММТ}

Таблииа 2

Parameters of MMT traffic flows

\begin{tabular}{|c|l|l|}
\hline $\begin{array}{c}\text { Пара- } \\
\text { метр }\end{array}$ & \multicolumn{1}{|c|}{ Описание } & $\begin{array}{l}\text { Допустимые } \\
\text { значения }\end{array}$ \\
\hline$\lambda_{k}$ & $\begin{array}{l}\text { Интенсивность трафика } \\
\text { от } k \text {-й беспроводной } \\
\text { станции, где } k=1, K\end{array}$ & $\begin{array}{l}1-6000 \text { [па- } \\
\text { кет/c] }\end{array}$ \\
\hline$l$ & Размер пакетов ММТ & $500-1500$ [Б] \\
\hline
\end{tabular}

\section{Архитектура разработанного исследовательского стенда}

Архитектура разработанного исследовательского стенда представлена на рисунке 2. В качестве аппаратных средств использован персональный компьютер с параметрами: процесcop - Intel Core i7 4700HQ 2400 - 3400 МГц, О3У - 16 Гб, ОС - Debian 10. Программный блок «Стенд моделирования» (СМ) включает модуль настройки (МН), описывающий пара- метры симуляции корпоративной БЛВС, и модуль генератора (МГ), осуществляющий генерацию входного трафика с экспоненциальным распределением времени интервалов поступления пакетов трафика ММТ. Программный блок СМ является программным модулем на языке $\mathrm{C}++$ для ns-3 [2] и содержит 396 строк.

Программный блок «Анализ результатов моделирования» (АРМ) включает: программный модуль (МП1) (написан на языке Python с использованием библиотеки pyshark [11], со-

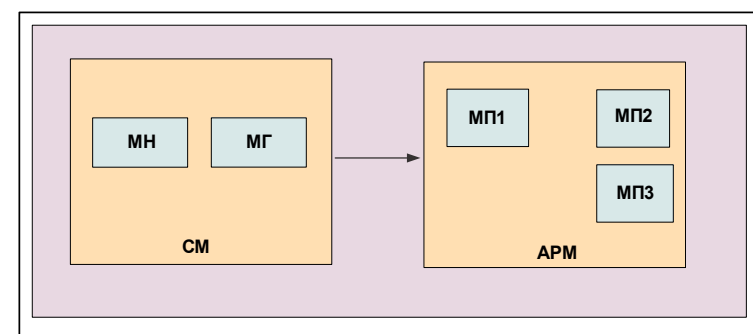

Рис. 2. Архитектура исследовательского стенда для получения и анализа вероятностно-временных характеристик корпоративной БЛВС

Fig. 2. The architecture of the research testbed for obtaining and analyzing the probabilistic time characteristics of the corporate WLAN 
держит 450 строк), позволяющий получить вероятностно-временные характеристики и расширенный набор функциональных характеристик корпоративной БЛВС; программный модуль (МП2) (написан на языках CL (WxMaxima [12], содержит 211 строк) и Python (содержит 388 строк)), который формирует и решает аналитические уравнения модели БЛВС; программный модуль (МП3) (написан на языке Python c использованием библиотек Numpy [13] и PyGnuplot [14], содержит 270 строк), который формирует результаты аналитического моделирования.

Программный блок СМ. Данный блок содержит два программных модуля (рис. 2) - МН и МГ. МН получает параметры из таблиц 1 и 2. Подробно разработка МН и МГ описана в работе [15], где приведены фрагменты программ на языке $\mathrm{C}++$. Рассмотрим временную диаграмму работы данного блока (рис. 3). На шагах $t 0$ и $t 1$ осуществляются запуск АР и STA $k$ $(k=1, K)$ и запуск перехвата трафика беспроводного интерфейса в РСАР-файл. МГ используется для запуска UDP-сервера (Server) на AP и $\mathrm{STA}_{1}$, настройки UDP-клиента (App) на беспроводных станциях $\operatorname{STA} k(k=1, K)$ (шаги $t 2$ и t3). После запуска UDP-сервера МГ включает отправку пакетов на UDP-клиентах (шаг $t 4)$. На рисунке 4 представлена временная диаграмма генератора, где $\theta_{i}$ - случайный интервал времени с экспоненциальным распределением $\lambda_{i}$, $n_{i}$ - номер отправляемого пакета ММТ; $l$ - размер пакета ММТ. По умолчанию буфер для пакета размером $l$ MMT равен 0 , перед отправкой в буфер добавляется номер пакета. На шаге $t 5$ точка доступа АР и каждая беспроводная стан-

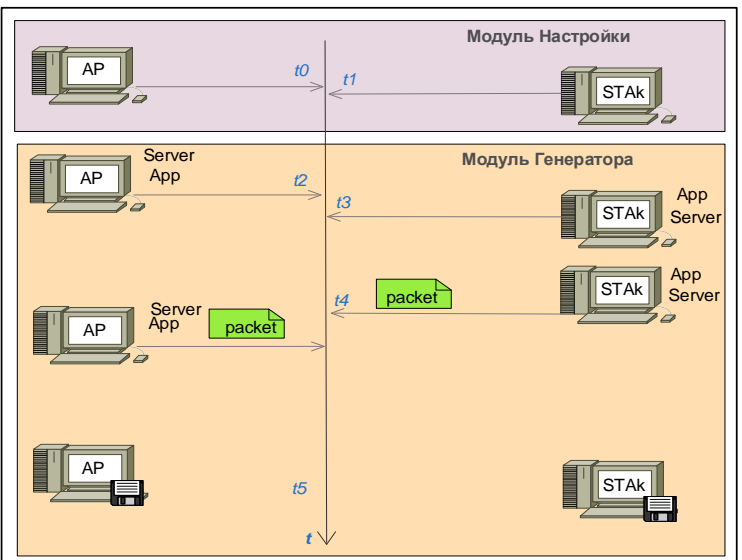

Рис. 3. Временная диаграмма работьл программного блока $C M$

Fig. 3. The timing diagram of the "Simulation testbed" software unit ция STA сохраняют данные о полученных (отправленных) пакетах в файл отчета, а также перехваченный трафик в РСАР-файл. Файл отчета содержит зависимость интенсивности доставленных пакетов $u_{C F}^{И}(\Lambda)$ от интенсивности трафика ММТ $\Lambda$ для БКС с протоколом $\mathrm{CF}$ $(C F=[D C F, P C F])$.

Программный блок АРМ представлен на рисунке 5. Программный модуль МП1 использует данные из РСАР-файла для получения зависимости:

- математического ожидания времени обслуживания пакета ММТ $T_{P C F}^{U_{P C}}(\Lambda)$ от интенсивности трафика ММТ $\Lambda$ для БКС с протоколом $\mathrm{CF}$, где $C F=(D C F, P C F)$;

- дисперсии времени обслуживания пакета $\sigma_{C F}^{2}(\Lambda)$ от интенсивности трафика ММТ $\Lambda$ для БКС с протоколом $\mathrm{CF}$, где $C F=(D C F$, $P C F)$;

- интенсивности обслуживания пакетов $\mu_{C F}(\Lambda)$ от интенсивности трафика ММТ $\Lambda$ для БКС с протоколом CF, где $C F=(D C F, P C F)$;

- коэффициента вариации $C_{C F}(\Lambda)$, выраженной соотношением

$$
C_{C F}(\Lambda)=100 \% * \sigma_{C F}(\Lambda) / T_{P C F}^{\mathrm{U}}(\Lambda) .
$$

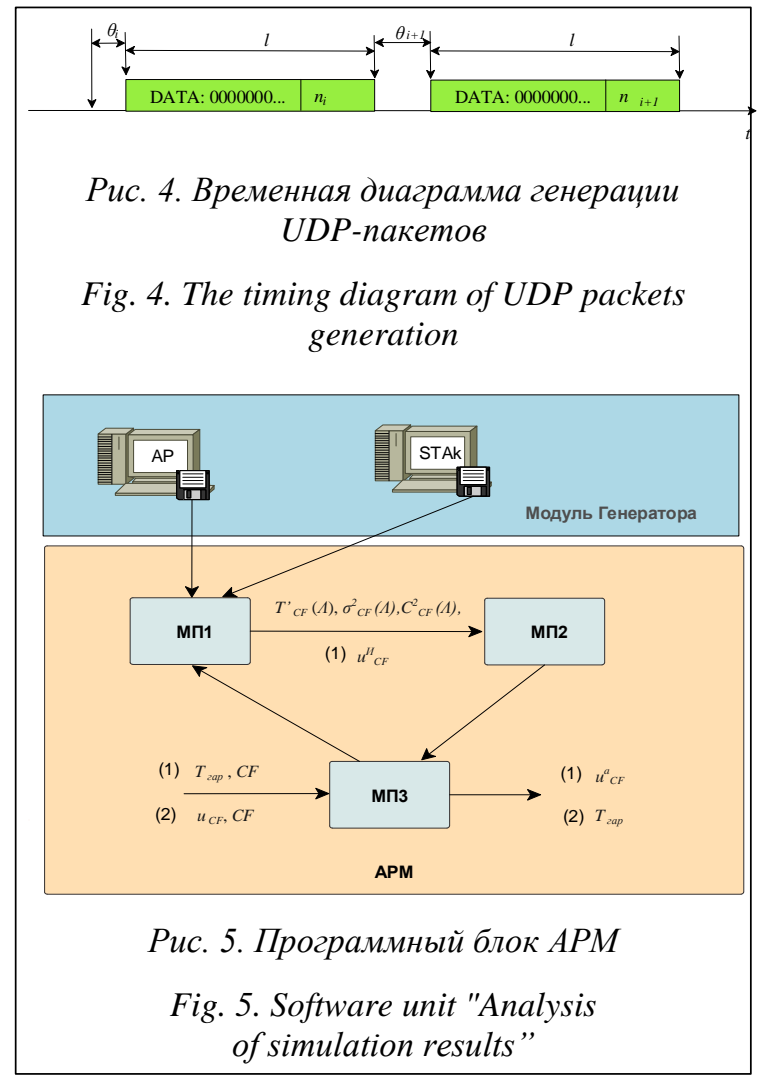

Для получения зависимости математического ожидания времени обслуживания пакета $T^{\mathrm{U}}{ }_{\mathrm{F}}(\Lambda)$ от интенсивности трафика MМТ $\Lambda$ 
блоком МП1 использует данные из РСАР-файла, полученного на шаге $t 5$ блоком СМ (рис. 3).

В РСАР-файле используется псевдозаголовок wlan_radio для записи метаданных кадра IEEE 802.11, в том числе таймера функции синхронизации времени TSF (Time Synchronization Function timer). Для определения времени отправки и получения кадра IEEE 802.11 используются метаданные начала кадра IEEE 802.11 TSF.start и окончания приема IEEE 802.11 TSF.end. Временная диаграмма для КП DCF и PCF представлена на рисунках 6 и 7. Для измерения времени обслуживания пакета ММТ используется значение $T^{\mathrm{U}_{i C F}}=T_{2}-T_{1}$.

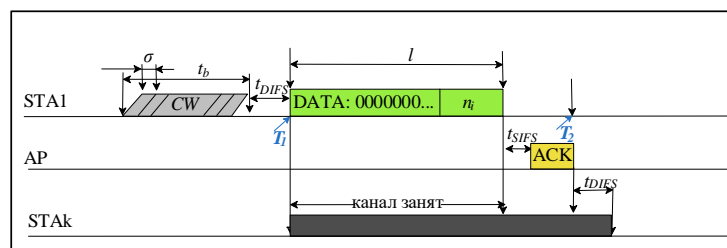

Рис. 6. Временная диаграмма для $C F=D C F$

Fig. 6. Timing diagram where $C F=D C F$

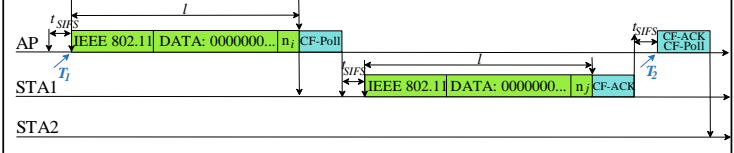

Рис. 7. Временная диаграмма для $C F=P C F$

Fig. 7. Timing diagram where $C F=P C F$

Программный модуль (МП2) формирует и решает следующие аналитические уравнения модели БЛВС [10]:

$$
\begin{gathered}
\hat{P}_{i \ni 1 I}=\frac{\alpha^{i} \gamma^{\frac{i-1}{2}}}{\sum_{i \ni 1 I} \alpha^{i} \gamma^{\frac{i-1}{2}}+\sum_{i \ni 2 I} \alpha^{i} \gamma^{\frac{i}{2}}}, \\
\hat{P}_{i \ni 1 I}=\frac{\alpha^{i} \gamma^{\frac{i}{2}}}{\sum_{i \ni 1 I} \alpha^{i} \gamma^{\frac{i-1}{2}}+\sum_{i \ni 2 I} \alpha^{i} \gamma^{\frac{i}{2}}},
\end{gathered}
$$

где подмножество $1 I$ индексов нечетных состояний и подмножество $2 I$ индексов четных состояний, а количество мест в очереди $s$ зависит от количества $K$ беспроводных станций, гаран-

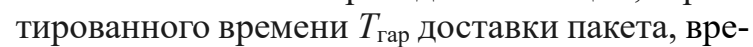
мени $T_{C F}$ канального протокола и принимается равным: $s=(K+1)\left(T_{\text {гар }} / T_{C F}\right)$.

Тогда аналитическая интенсивность $u_{C F}^{a}$ доставленных пакетов вычисляется следующим образом: $u_{C F}^{a}=\Lambda\left(1-P_{s+1}\right)$, а аналитическая интенсивность $u_{C F}^{a}$ равна:

$$
\begin{aligned}
& u_{C} F^{a}=\Lambda\left(1-\frac{\alpha^{i} \gamma^{\frac{i-1}{2}}}{\sum_{i \ni 1 I} \alpha^{i} \gamma^{\frac{i-1}{2}}+\sum_{i \ni 2 I} \alpha^{i} \gamma^{\frac{i}{2}}}\right)+ \\
& +\Lambda\left(1-\frac{\alpha^{i} \gamma^{\frac{i}{2}}}{\sum_{i \ni 1 I} \alpha^{i} \gamma^{\frac{i-1}{2}}+\sum_{i \ni 2 I} \alpha^{i} \gamma^{\frac{i}{2}}}\right) .
\end{aligned}
$$

Время доставки пакета МMT $T^{\phi}$ сF с использованием КП CF, где $C F=(D C F, P C F)$, учитывающее время в очереди и время обслуживания, определяется соотношением

\section{$T^{\phi}{ }_{C F}=n / u^{a}{ }_{C F}$.}

МПЗ формирует результаты аналитического моделирования в соответствии с задачей «Для заданного времени $T_{\text {гар }}$ гарантированной доставки пакетов, для БКС, использующих КП CF, определить предельную интенсивность $u_{C F}^{a}$ доставленных протоколов $\mathrm{CF}$, где $C F=$ $=(D C F, P C F) »$.

\section{Реализация исследовательского стенда}

Рассмотрим пример работы стенда для получения и анализа вероятностно-временных характеристик корпоративной БЛВС при ограничениях: «Для заданного времени гарантированной доставки пакетов $T_{\text {гар }}=0,001 \mathrm{c}$, для БКС, использующих КП СF, $C F=(D C F, P C F)$, определить предельную интенсивность $u_{C F}^{a}$ доставленных пакетов. Сравнить предельную интенсивность $u_{D C F}^{a}$ и $u^{a}{ }_{P C F} \gg$. Параметры исследуемой БЛВС приведены в таблице 3 .

Для получения суммарной интенсивности входного трафика необходимо провести исследование корпоративной БЛВС с параметрами из таблицы 3 , используя блок СМ. С помощью программных модулей МП1 и МП2 получены следующие зависимости для двух канальных протоколов $C F=(D C F, P C F)$ : математическое ожидание времени обслуживания пакета ММТ $T^{\mathrm{U}}{ }_{D C F}(\Lambda), T^{\mathrm{h}}{ }_{P C F}(\Lambda)$, дисперсия времени обслуживания пакета MMT $\sigma^{2} D C F(\Lambda), \sigma^{2} P C F(\Lambda)$, коэффициент вариации времени обслуживания пакета MMT $C_{D C F}(\Lambda), C_{P C F}(\Lambda)$, интенсивность обслуживания пакетов $\mu_{D C F}(\Lambda), \mu_{P C F}(\Lambda)$ для расчета суммарной интенсивности входного трафика ММТ. Результат работы блока МП2 зависимости времени доставки пакета ММТ $T^{\Phi}{ }_{D C F}(\Lambda)$ от интенсивности трафика ММТ для $C F=(D C F, P C F)$ представлены на рисунке 8 . $\mathrm{C}$ помощью программных блоков МП2 и МП3 получена зависимость вероятности отказа в об- 
Параметры исследуемой БЛВС

Таблииа 3

Parameters of the WLAN of interest

Table 3

\begin{tabular}{|c|c|c|c|}
\hline Параметр & $\begin{array}{c}\text { Допустимые } \\
\text { значения }\end{array}$ & \multicolumn{2}{|c|}{$\mathrm{CF}=\mathrm{PCF}$} \\
\hline dot11BeaconPeriod & $1 . .65535[\mathrm{TU}]$ & dot11DTIMPeriod & 1 [dot11BeaconPeriod] \\
\hline$h w \_$mode & $g$ & $\begin{array}{l}\text { dot11MediumOccupancy } \\
\text { Limit }\end{array}$ & 1000 [TU] \\
\hline basic_rates & 540 [100 kbps] & dot11CFPPeriod & 1 [dot11DTIMPeriod] \\
\hline$K$ & $1-25$ & \multicolumn{2}{|c|}{ Параметры потоков трафика ММТ } \\
\hline \multicolumn{2}{|l|}{ CF $=$ DCF } & $\lambda_{k}$ & 1-6 000 [пакет/c] \\
\hline dot11DTIMPeriod & $l$ & $500-1500[$ [Б] & \\
\hline dot11MediumOccupancyLimit & $0[\mathrm{TU}]$ & \multirow{2}{*}{\multicolumn{2}{|c|}{+2}} \\
\hline dot11CFPPeriod & 255 [dot11DTIMPeriod] & & \\
\hline
\end{tabular}

служивании пакета ММТ для $C F=[D C F, P C F]$ (рис. 9). Полученная зависимость (рис. 8) позволяет определить входную интенсивность трафика ММТ для $T_{\text {гар }}=0,001 \mathrm{c}$, а именно $\Lambda_{D C F} \approx 3100$ пакет/c, а $\Lambda_{P C F} \approx 1500$ пакет/c, при $\mu_{D C F} \approx 3160$ пакет/с и $\mu_{P C F}(\Lambda) \approx 2074$ пакет/c. Отсюда $\alpha_{D C F} \approx 0,98$ и $\alpha_{P C F} \approx 0,72$. Полученные зависимости (рис. 9) позволяют установить $P^{D C{ }_{s+1}} \approx 0,01$ и $P^{P C F}{ }_{s+1} \approx 0,02$ и решение задачи - предельную интенсивность $u_{C F}^{a}$ доставленных пакетов ММТ: $u^{a}{ }_{D C F} \approx 3069$ пакет/с и $u_{\text {PCF }}^{a} \approx 2033$ пакет/с. Полученные результаты позволяют сравнить предельные интенсивности доставленных пакетов трафика ММТ для КП DCF и РCF. Для заданных в задаче параметров рекомендуется использовать КП DCF.

\section{Заключение}

В статье представлены архитектура и программная реализация разработанного стенда. Подробно описаны блоки стенда для исследования корпоративной БЛВС с помощью дискретно-событийного моделирования, оценки и анализа результатов путем аналитических расчетных соотношений, полученных авторами. В статье содержатся результаты исследования вероятностно-временных характеристик на примере корпоративной БЛВС с заданной конфигурацией для двух КП БКС. Функциональность разработанного стенда обеспечивает возможность работы в двух режимах. В режиме разработки новой БЛВС, когда известными параметрами являются паспортные данные оборудования, логические характеристики протоколов и предполагаемые характеристики трафика, используется полный набор функциональных модулей и блоков, чтобы обеспечить согласование трафика с ресурсами передачи и обработки и заданную производительность БЛВС. В режиме эксплуатации, когда мониторинг обеспечивает реальные характеристики трафика и протоколов, администратор БЛВС получает возможность оценить производитель-

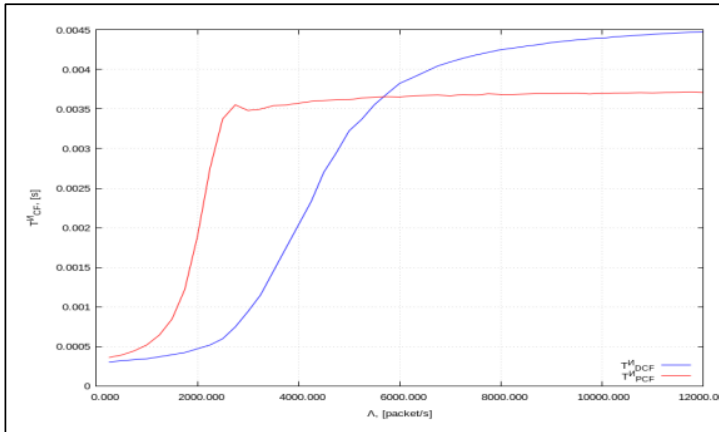

Рис. 8. Зависимость времени доставки пакета $T^{\phi}{ }_{C F}$ (И) от интенсивности трафика ММT

Fig. 8. Dependence of the packet delivery time $T^{\phi}{ }_{C F}(\Lambda)$ on the multimedia traffic intensity

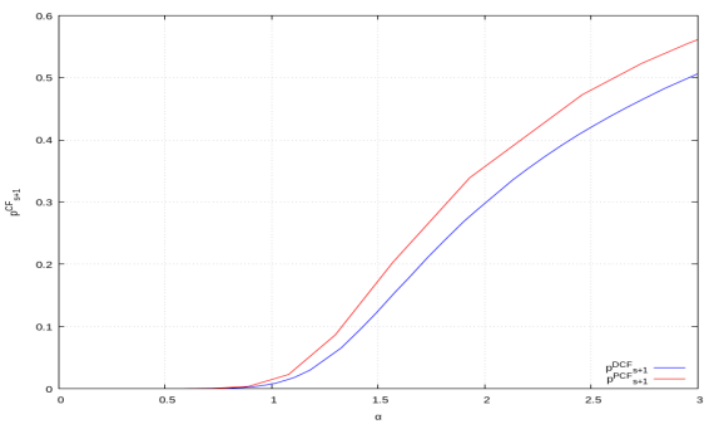

Pис. 9. Зависимость вероятности $P_{s+1}$ отказа обслуживания пакетов от загруженности БКС $\alpha$

Fig. 9. Dependence of the deny of service probability $P_{s+1}$ on the wireless channel load $\alpha$ 
ность БЛВС и интенсивность трафика - используется ограниченный набор модулей, который требует гораздо меньше времени для оценки производительности БЛВС и дает возможность изменять настройки и обеспечивать адаптивное регулирование, соответствующее требованиям QoS.
Используя разработанный программный комплекс, авторы проводят работы по исследованию вероятностно-временных характеристик сегментов БЛВС, функционирующих в составе Информационной вычислительной сети Национального исследовательского университета «МЭИ».

\title{
Лumepamypa
}

1. Dujovne D., Turletti T. and Filali F. A taxonomy of IEEE 802.11 wireless parameters and open source measurement tools. IEEE Communications Surveys \& Tutorials, 2010, vol. 12, no. 2, pp. 249-262. DOI: 10.1109/SURV.2010.021110.00020.

2. Ns-3: Network Simulator. URL: https://www.nsnam.org/ (дата обращения: 21.09.2020).

3. Yin Y., Gao Y., Hei X. Performance evaluation of a unified IEEE 802.11 DCF model in NS-3. Proc. SIMUTools Conf., 2019, pp. 395-406. DOI: 10.1007/978-3-030-32216-8_38.

4. Deutsch P., Veytser L., Cheng B. LL SimpleWireless: A controlled MAC/PHY wireless model to enable network protocol research. Proc. Workshop on NS3 (WNS3), 2016, pp. 71-78. DOI: 10.1145/2915371.2915376.

5. Tian Le, Deronne S., Latré S., Jeroen F. Implementation and validation of an IEEE 802.11ah module for NS-3. Proc. Workshop on NS3 (WNS3), 2016, pp. 49-56. DOI: 10.1145/2915371.2915372.

6. Assasa H., Widmer J. Implementation and evaluation of a WLAN IEEE 802.11ad model in ns-3. Proc. WNS3, 2016, pp. 57-64. DOI: 10.1145/2915371.2915377.

7. Safavi-Naeini H.-A., Nadeem F., Roy S. Investigation and improvements to the OFDM Wi-Fi physical layer abstraction in ns-3. Proc. WNS3, 2016, pp. 65-70. DOI: 10.1145/2915371.2915387.

8. IEEE Standard for Information Technology: Telecommunications and information exchange between systems local and metropolitan area networks: Specific requirements. Pt. 11, Wireless LAN MAC and PHY Specifications. DOI: 10.1109/IEEESTD.2016.7786995.

9. Hostapd: IEEE 802.11 AP, W1.Fi. URL: https://www.w1.fi/cgit/hostap/plain/hostapd/hostapd.conf. (дата обращения: 21.09.2020).

10. Абросимов Л.И., Руденкова М.А., Хаю Х. Методика определения гарантированной доставки трафика в корпоративных беспроводных локальных вычислительных сетях // Вестн. Воронежского гос. технич. ун-та. 2020. Т. 16. № 5. С. 38-49. DOI: 10.36622/VSTU.2020.16.5.006.

11. Pyshark 0.4.2.11. URL: https://pypi.org/project/pyshark/ (дата обращения: 21.09.2020).

12. "Wxmaxima". Wxmaxima-Developers.Github.Io, 2020, URL: https://wxmaxima-developers.github.io/ wxmaxima/ (дата обращения: 21.09.2020).

13. Numpy v1.19.0. URL: https://numpy.org/ (дата обращения: 03.10.2020).

14. Pygnuplot 0.11.16. URL: https://pypi.org/project/PyGnuplot/ (дата обращения: 03.10.2020).

15. Rudenkova M. A methodology of modeling the IEEE 802.11 wireless LAN using ns-3. Proc. INFORINO, 2020, pp. 1-4. DOI: 10.1109/Inforino48376.2020.9111782.

\section{Architecture and programming implementation of research testbed of a corporate Wireless Local Area Network}

\author{
L.I. Abrosimov ${ }^{1}$, Dr.Sc. (Engineering), Professor, AbrosimovLI@mpei.ru \\ M.A. Orlova ${ }^{1}$, Assistant, OrlovaMA@mpei.ru \\ H. Khayou ${ }^{1}$, Postgraduate Student, hussein.khayou@gmail.com \\ ${ }^{1}$ National Research University "Moscow Power Engineering Institute”, \\ Moscow, 111250, Russian Federation
}

Abstract. The paper presents the architecture and implementation of a research testbed for obtaining and analyzing the probabilistic time characteristics of a corporate Wireless Local Area Network (WLAN). To develop this testbed, the authors obtained mathematical relations for calculating the guaranteed intensity of multimedia traffic.

The research testbed architecture includes the two independent blocks. The block "Simulation testbed" contains the corporate WLAN description and the multimedia traffic flows in the discrete-event simulation system ns-3. The block "Analyzing simulation results" contains programs for analyzing files of transmitted traffic and simulation results and programs for calculating performance characteristics. To write the block 
"Analyzing simulation results ", the authors used the Python3 language, the analysis of the transmitted traffic files was performed using the pyshark library.

The paper also contains the analytical equations of the WLAN model used in the block " Analyzing simulation results ". The above equations allow us to determine the maximum intensity of the delivered packets for a prescribed time of guaranteed packet delivery, for wireless communication channels using a prescribed channel protocol. A software implementation of the research testbed affords the opportunity to get the dependence of intensity guaranteed multimedia traffic for the specified parameters: structure WLAN, the settings of the wireless communication channel protocols, and channel access control.

The developed testbed provides the capability of operation in two modes. In the development mode of a new WLAN, when the known parameters are the equipment passport data, logical characteristics of protocols, and expected traffic characteristics, a full set of functional modules and blocks is used, which allows both traffic matching with transmission and processing resources, and the specified performance of the WLAN. In operation mode, when monitoring allows you to get actual characteristics of traffic and protocols, the testbed allows the WLAN administrator to test the performance of the WLAN and the traffic intensity. This mode uses a limited module set, which requires much less time to evaluate the performance of the WLAN, provides the ability to adaptively change the settings of the WLAN, and provides the performance characteristics of the WLAN that meet the requirements of QoS.

Keywords: network simulator software, research testbed architecture, WLAN, performance, media access protocol.

\section{References}

1. Dujovne D., Turletti T. and Filali F. A taxonomy of IEEE 802.11 wireless parameters and open source measurement tools. IEEE Communications Surveys \& Tutorials, 2010, vol. 12, no. 2, pp. 249-262. DOI: 10.1109/SURV. 2010.021110 .000200 .

2. Ns-3: Network Simulator. Available at: https://www.nsnam.org/ (accessed September 21, 2020).

3. Yin Y., Gao Y., Hei X. Performance evaluation of a unified IEEE 802.11 DCF model in NS-3. Proc. SIMUTools Conf., 2019, pp. 395-406. DOI: 10.1007/978-3-030-32216-8_38.

4. Deutsch P., Veytser L., Cheng B. LL SimpleWireless: A controlled MAC/PHY wireless model to enable network protocol research. Proc. WNS3, 2016, pp. 71-78. DOI: 10.1145/2915371.2915376.

5. Tian Le, Deronne S., Latré S., Jeroen F. Implementation and validation of an IEEE 802.11ah module for NS-3. Proc. Workshop on NS3 (WNS3), 2016, pp. 49-56. DOI: 10.1145/2915371.2915372.

6. Assasa H., Widmer J. Implementation and evaluation of a WLAN IEEE 802.11ad model in ns-3. Proc. WNS3, 2016, pp. 57-64. DOI: 10.1145/2915371.2915377.

7. Safavi-Naeini H.-A., Nadeem F., Roy S. Investigation and improvements to the OFDM Wi-Fi physical layer abstraction in ns-3. Proc. WNS3, 2016, pp. 65-70. DOI: 10.1145/2915371.2915387.

8. IEEE Standard for information technology: Telecommunications and information exchange between systems local and metropolitan area networks: Specific requirements. Pt. 11, Wireless LAN MAC and PHY Specifications. DOI: 10.1109/IEEESTD.2016.7786995.

9. Hostapd: IEEE 802.11 AP, W1.Fi. Available at: https://www.w1.fi/cgit/hostap/plain/hostapd/hostapd.conf. (accessed: 21.09.2020).

10. Abrosimov L.I., Rudenkova M.A., Khayou H. Methodology to determine guaranteed delivery traffic rate in corporate wlan. The Bulletin of Voronezh State Technical University, 2020, vol. 16, no. 5, pp. 38-49 (in Russ.). DOI: 10.36622/VSTU.2020.16.5.006.

11. Pyshark 0.4.2.11. Available at: https://pypi.org/project/pyshark/ (accessed September 09, 2020).

12. "Wxmaxima". Wxmaxima-Developers.Github.Io, 2020. Available at: https://wxmaxima-developers.github. io/wxmaxima/ (accessed September 09, 2020).

13. Numpy v1.19.0. Available at: https://numpy.org/ (accessed October 03, 2020).

14. Pygnuplot 0.11.16. Available at: https://pypi.org/project/PyGnuplot/ (accessed October 03, 2020).

15. Rudenkova M. A Methodology of modeling the IEEE 802.11 wireless LAN using ns-3. Proc. INFORINO, 2020, pp. 1-4. DOI: 10.1109/Inforino48376.2020.9111782.

\section{Для цитирования}

Абросимов М.И., Орлова М.А., Хаю Х. Архитектура и программная реализация исследовательского стенда корпоративной беспроводной мокальной вычислительной сети// Программные продукты и системы. 2021. Т. 34. № 1. C. 172-179. DOI: 10.15827/0236-235X.133.172-179.

\section{For citation}

Abrosimov L.I., Orlova M.A., Khayou H. Architecture and programming implementation of research testbed of a corporate Wireless Local Area Network. Software \& Systems, 2021, vol. 34, no. 1, pp. 172-179 (in Russ.). DOI: 10.15827/0236-235X.133.172-179. 